\title{
Le milieu scolaire québécois face aux défis de l'accueil des élèves réfugiés : quels enjeux pour la gouvernance scolaire et la formation des intervenants scolaires?
}

\section{The Quebec school sector facing the challenges of receiving refugee students: what are the issues for school governance and training school workers? \\ El medio escolar quebequense ante los retos que comporta la acogida de alumnos refugiados: ¿cuáles son los desafíos para la gobernanza escolar y la formación de los intervinientes escolares?}

\author{
Garine Papazian-Zohrabian, Caterina Mamprin, Vanessa Lemire, Alyssa \\ Turpin-Samson, Ghayda Hassan, Cécile Rousseau et Ray Aoun
}

\section{Volume 46, numéro 2, automne 2018}

Pluralisme, équité et rapports ethniques dans la formation du personnel des milieux éducatifs

URI : https://id.erudit.org/iderudit/1055569ar

DOI : https://doi.org/10.7202/1055569ar

Aller au sommaire du numéro

Éditeur(s)

Association canadienne d'éducation de langue française

ISSN

1916-8659 (numérique)

Découvrir la revue

\section{Citer cet article}

Papazian-Zohrabian, G., Mamprin, C., Lemire, V., Turpin-Samson, A., Hassan, G., Rousseau, C. \& Aoun, R. (2018). Le milieu scolaire québécois face aux défis de l'accueil des élèves réfugiés : quels enjeux pour la gouvernance scolaire et la formation des intervenants scolaires? Éducation et francophonie, 46(2),

208-229. https://doi.org/10.7202/1055569ar

\section{Résumé de l'article}

En 2015 et en 2016, le Québec accueille plus de 7583 réfugiés syriens. À la suite de cette arrivée importante, plusieurs écoles se mobilisent pour faciliter l'accueil des nouveaux élèves. Dans ce contexte psychosocial particulier et grâce à une subvention du Conseil de recherches en sciences humaines du Canada, nous menons une recherche-action intitulée Favoriser l'intégration sociale et scolaire des élèves réfugiés syriens en développant leur sentiment d'appartenance à l'école, leur bien-être psychologique et celui de leurs familles. Cet article reprend certains résultats de cette recherche menée dans trois écoles de la région montréalaise auprès d'élèves syriens de cinq classes d'accueil du primaire et du secondaire. Les données découlent de groupes de parole menés avec les élèves sur des thèmes sensibles (p. ex., la migration, la différence, la mort), d'entrevues de groupe avec le personnel enseignant des classes d'accueil et d'entrevues individuelles avec les directions adjointes des écoles participantes. La documentation de l'implantation de cette action et de son influence sur le développement du bien-être et du sentiment d'appartenance des élèves permet de mettre en relief un certain mal-être des jeunes, des difficultés d'intégration socioscolaire, un manque de ressources humaines et matérielles, ainsi qu'un besoin en formation des divers acteurs scolaires. Les résultats de la recherche permettent de dégager plusieurs enjeux relatifs à la gouvernance et à la formation des acteurs scolaires.
Tous droits réservés () Association canadienne d'éducation de langue française, 2018
Cecument est protégé par la loi sur le droit d'auteur. L'utilisation des services d’Érudit (y compris la reproduction) est assujettie à sa politique d'utilisation que vous pouvez consulter en ligne. 


\section{Le milieu scolaire québécois face aux défis de l'accueil des élèves réfugiés: quels enjeux pour la gouvernance scolaire et la formation des intervenants scolaires?}

Citer cet article :

PAPAZIAN-ZOHRABIAN, G., MAMPRIN, C., LEMIRE, V., TURPINSAMSON, A., HASSAN, G. et ROUSSEAU, C. (2018). Le milieu scolaire québécois face aux défis de l'accueil des élèves réfugiés: quels enjeux pour la gouvernance scolaire et la formation des

intervenants scolaires? Éducation et francophonie, 46(2), 208-229.

\section{Garine PAPAZIAN-ZOHRABIAN}

Université de Montréal, Québec, Canada

\section{Caterina MAMPRIN}

Université de Montréal, Québec, Canada

Vanessa LEMIRE

Université de Montréal, Québec, Canada

\section{Alyssa TURPIN-SAMSON}

Université de Montréal, Québec, Canada

\section{Ghayda HASSAN}

Université du Québec à Montréal, Québec, Canada

\section{Cécile ROUSSEAU}

Université McGill, Québec, Canada

Ray AOUN

Université de Montréal, Québec, Canada 


\section{RÉSUMÉ}

En 2015 et en 2016, le Québec accueille plus de 7583 réfugiés syriens. À la suite de cette arrivée importante, plusieurs écoles se mobilisent pour faciliter l'accueil des nouveaux élèves. Dans ce contexte psychosocial particulier et grâce à une subvention du Conseil de recherches en sciences humaines du Canada, nous menons une recherche-action intitulée Favoriser l'intégration sociale et scolaire des élèves réfugiés syriens en développant leur sentiment d'appartenance à l'école, leur bien-être psychologique et celui de leurs familles. Cet article reprend certains résultats de cette recherche menée dans trois écoles de la région montréalaise auprès d'élèves syriens de cinq classes d'accueil du primaire et du secondaire. Les données découlent de groupes de parole menés avec les élèves sur des thèmes sensibles (p. ex., la migration, la différence, la mort), d'entrevues de groupe avec le personnel enseignant des classes d'accueil et d'entrevues individuelles avec les directions adjointes des écoles participantes. La documentation de l'implantation de cette action et de son influence sur le développement du bien-être et du sentiment d'appartenance des élèves permet de mettre en relief un certain mal-être des jeunes, des difficultés d'intégration socioscolaire, un manque de ressources humaines et matérielles, ainsi qu'un besoin en formation des divers acteurs scolaires. Les résultats de la recherche permettent de dégager plusieurs enjeux relatifs à la gouvernance et à la formation des acteurs scolaires.

ABSTRACT

\section{The Quebec school sector facing the challenges of receiving refugee students: what are the issues for school governance and training school workers?}

Garine PAPAZIAN-ZOHRABIAN, University of Montreal, Quebec, Canada

Caterina MAMPRIN, University of Montreal, Quebec, Canada

Vanessa LEMIRE, University of Montreal, Quebec, Canada

Alyssa TURPIN-SAMSON, University of Montreal, Quebec, Canada

Ghayda HASSAN, University of Quebec in Montreal, Quebec, Canada

Cécile ROUSSEAU, McGill University, Quebec, Canadaa

Ray AOUN, University of Montreal, Quebec, Canada

In 2015 and 2016, Quebec welcomed more than 7,583 Syrian refugees. In the wake of this major arrival, several schools mobilized to facilitate the reception of new students. In this particular psychosocial context and with a grant from the Social Sciences and Humanities Research Council of Canada, we conducted an action-research entitled Fostering the social and scholastic integration of Syrian refugee students by developing their sense of belonging to the school, their psychological well-being and that of their families. This article presents some results from this research, which was conducted in three schools in the Montreal area with Syrian students from five 
Le milieu scolaire québécois face aux défis de l'accueil des élèves réfugiés:

primary and secondary school classes. The data is derived from discussion groups with students on sensitive topics (eg, migration, differences, death), group interviews with reception class teachers, and individual interviews with vice-principals of the participating schools. The documentation on the implementation of this action and how it influenced the development of a sense of well-being and belonging for the students reveals a certain malaise among these youngsters, problems with socio-scholastic integration, a lack of human and material resources, and a need to provide school workers with more training. The results of the research identify several issues related to governance and the training of school stakeholders.

\title{
RESUMEN
}

\section{El medio escolar quebequense ante los retos que comporta la acogida de alumnos refugiados: ¿cuáles son los desafíos para la gobernanza escolar y la formación de los intervinientes escolares?}

\author{
Garine PAPAZIAN-ZOHRABIAN, Universidad de Montreal, Quebec, Canadá \\ Caterina MAMPRIN, Universidad de Montreal, Quebec, Canadá \\ Vanessa LEMIRE, Universidad de Montreal, Quebec, Canadá \\ Alyssa TURPIN-SAMSON, Universidad de Montreal, Quebec, Canadá \\ Ghayda HASSAN, Universidad de Quebec en Montreal, Quebec, Canadá \\ Cécile ROUSSEAU, Universidad McGill, Quebec, Canadá \\ Ray AOUN, Universidad de Montreal, Quebec, Canadá
}

En 2015 y en 2016, Quebec recibió más de 7583 refugiados sirios. Como consecuencia de esta importante afluencia, varias escuelas se movilizaron para facilitar la recepción de los nuevos alumnos. En este contexto psicosocial particular y gracias a una subvención del Consejo de investigaciones en ciencias humanas de Canadá, hemos realizado una investigación-acción intitulada Favorecer la integración social y escolar de los alumnos refugiados sirios en el desarrollo de su sentimiento de pertenencia a la escuela, su bienestar psicosocial y el de sus familias. Este artículo retoma algunos de los resultados de dicha investigación, realizada en tres escuelas de la región montrealesa entre alumnos sirios de cinco clases de acogida de primaria y secundaria. Los datos provienen de grupos de discusión realizados con los alumnos sobre temas sensibles (por ejemplo, la migración, la diferencia, la muerte), entrevistas de grupo con personal docente de las clases de acogida y entrevistas individuales con las direcciones adjuntas de las escuelas participantes. La documentación de la implantación de esta acción y de su influencia sobre el desarrollo del bienestar y del sentimiento de pertenencia de los alumnos permite poner de relieve un cierto malestar de los jóvenes, de las dificultades de integración socio-escolar, de una falta de recursos humanos y materiales, así como una necesidad de formación de los diversos actores escolares. Los resultados de la investigación permiten identificar varios retos relacionados con la gobernanza y la formación de los actores escolares. 


\section{INTRODUCTION}

Depuis 2011, le conflit syrien a provoqué le déplacement de près de 13,5 millions d'individus. En 2016, le Canada a accueilli plus de 46700 réfugiés, dont $47 \%$ sont des mineurs (Haut Commissariat des Nations Unies pour les réfugiés, Canada, 2017). Selon le ministère de l'Immigration, de la Diversité et de l'Inclusion (2017), le Québec a accueilli 7583 réfugiés syriens en 2015-2016. À la suite de cette arrivée importante, de nombreuses écoles québécoises se sont mobilisées pour recevoir ces élèves, comme elles l'avaient fait dans le passé (Kirk, 2002).

Dans ce contexte, nous avons mené une recherche-action intitulée Favoriser l'intégration sociale et scolaire des élèves réfugiés syriens en développant leur sentiment d'appartenance à l'école, leur bien-être psychologique et celui de leurs familles (Conseil de recherches en sciences humaines du Canada, 2016-2017) pour évaluer l'influence d'une action scolaire visant l'intégration socioscolaire des jeunes réfugiés syriens.

Le point de départ de notre réflexion a été la situation psychosociale des réfugiés, les conditions d'adversité dans lesquelles ils ont survécu et aussi le potentiel de mal-être dans lequel ils se trouvent à leur arrivée. Cet article présente les résultats de cette recherche, qui soulignent l'importance du développement du bien-être psychologique et du sentiment d'appartenance à l'école des élèves réfugiés, ainsi que le rôle essentiel de l'école dans leur accueil. Entre autres, nous aborderons certains éléments systémiques et contextuels liés à l'accueil, et nous discuterons de certains enjeux liés à la gouvernance scolaire et à la formation du personnel enseignant.

\section{PROBLÉMATIQUE}

\section{Situation des élèves réfugiés}

Le Québec a accueilli de nombreux jeunes réfugiés syriens qui ont souffert de multiples violations de leurs droits fondamentaux: massacres, assassinats, exécutions, enlèvements, prises d'otage, torture, violences sexuelles, recrutement d'enfants par les forces armées (Hassan et al., 2016). À cause de cette exposition, ces enfants sont susceptibles d'éprouver des problèmes de santé mentale, résultant à la fois de leurs expériences liées à la guerre et des déplacements qui en ont découlé (Sirin et RogersSirin, 2015). Chez les enfants, ce mal-être se traduit par des troubles du sommeil, des symptômes anxieux, dépressifs et somatiques, des comportements agressifs ou encore des symptômes post-traumatiques. Certaines de leurs facultés cognitives peuvent également être affectées (Hassan et al., 2016; Özer, irin et Oppedal, 2013).

Peu de recherches abordent la situation psychosociale des réfugiés syriens réinstallés au Québec, mais plusieurs ont documenté ces problématiques chez d'autres groupes de réfugiés. Selon l'Organisation mondiale de la Santé (2016, citée dans Silove, 
Ventevogel et Rees, 2017), le taux de personnes traumatisées parmi les réfugiés (15\%) est de loin supérieur à celui d'une population de non-réfugiés $(1,1 \%)$. Les études montrent aussi que mise à part l'adversité de la vie prémigratoire, la santé mentale des familles réfugiées réinstallées est influencée par les pertes et les deuils migratoires, les différences culturelles et linguistiques, et les défis économiques (Kanouté et Lafortune, 2011; Miller et Rasmussen, 2010; 2017, cités dans Silove, Ventevogel et Rees, 2017; Pacione et al., 2012). Bien qu'un grand nombre d'enfants réfugiés soient résilients, de nombreux autres souffrent de problèmes aussi bien internalisés, tels que l'anxiété ou la dépression, qu'externalisés, tels que les difficultés de comportement et de concentration (Hart, 2009), même si ces symptômes se conjuguent avec des forces et des stratégies de survie (Rutter, 2003).

À l'instar des élèves immigrants, plusieurs défis attendent les élèves réfugiés, comme l'adaptation aux attentes scolaires et aux différences culturelles du pays d'accueil, et l'apprentissage d'une nouvelle langue (Kanouté, Gosselin-Gagné, Guennouni Hassani et Girard, 2016; Kupzyk, Banks et Chadwell, 2016; Tyrer et Fazel, 2014). En s'appuyant sur les données de l'Organisation de coopération et de développement économiques (2012, PISA2), Sarot et Moro (2016) soulignent que les élèves issus de l'immigration sont deux fois plus susceptibles de compter parmi les élèves en difficulté. Cette probabilité peut augmenter chez les réfugiés qui, à la suite de deuils et de traumatismes, peuvent avoir des difficultés d'adaptation et d'apprentissage scolaires (Papazian-Zohrabian, 2015, 2016).

En plus de ces difficultés scolaires, les élèves réfugiés peuvent vivre de la discrimination dans leur pays d'accueil, ce qui peut engendrer des conséquences sérieuses sur leur santé mentale (Beiser et Hou, 2016) et sur leur adaptation sociale (Montgomery et Foldspang, 2008).

Correa-Velez, Gifford et Barnett (2010) soulignent que le bien-être de ces élèves est lié à l'environnement social et à l'inclusion proposée par la société d'accueil. Le sentiment d'appartenance est l'un des meilleurs prédicteurs du bien-être chez les enfants dans les premières années suivant l'installation dans le pays d'accueil (Correa-Velez et al., 2010). Chez ces adolescentes et adolescents, l'expérience scolaire et le sentiment d'appartenance peuvent avoir une influence sur le bien-être général ainsi que sur l'adaptation au pays d'accueil (Kia-Keating et Ellis, 2007).

Par conséquent, il importe de favoriser une culture scolaire sécurisante pour les élèves, un milieu moins stressant ainsi qu'un modèle relationnel favorisant les liens intrascolaires (Bartlett, Mendenhall et Ghaffar-Kucher, 2017). 


\section{Rôle de l'école}

Persson et Rousseau (2008) ainsi que Kirk (2002) accordent une place centrale à l'école dans sa contribution au développement du bien-être psychologique des enfants traumatisés par la guerre. L'école est d'ailleurs centrale dans le modèle systémique d'intervention éducative destiné aux enfants réfugiés de Hamilton et Moore (2004), qui vise le développement du bien-être émotionnel et la réussite scolaire de ces enfants à travers une intervention permettant l'expression symbolique et touchant les divers systèmes dans lesquels ils évoluent.

Yohani (2010) relève toutefois la présence, dans les milieux scolaires, de plusieurs lacunes pouvant freiner le développement du bien-être des enfants réfugiés, comme le manque de formation et de ressources pour le personnel enseignant. McNeely et ses collaborateurs (2017) soulignent l'importance d'évaluer les programmes d'enseignement pour les nouveaux arrivants et de participer au développement professionnel du personnel enseignant grâce à des formations et à des activités de soutien. Le manque de formation des milieux scolaires concernant le vécu des élèves réfugiés peut engendrer des pratiques discriminatoires (Stewart, 2011) ainsi qu'une mauvaise compréhension du vécu des familles et des difficultés scolaires des élèves (Tyrer et Fazel, 2014).

Ainsi, l'école peut être considérée comme un espace important pour favoriser l'accueil et l'inclusion sociale des jeunes réfugiés. Toutefois, peu de recherches sont menées sur les moyens à offrir aux écoles pour qu'elles puissent soutenir les élèves et leurs familles (Block, Cross, Riggs et Gibbs, 2014). C'est pourquoi notre recherche-action vise, entre autres, le développement de nouvelles pratiques scolaires d'accueil favorisant le bien-être psychologique des jeunes réfugiés ainsi que leur sentiment d'appartenance à l'école.

\section{CADRE DE RÉFÉRENCE}

\section{Bien-être psychologique}

Le concept de bien-être se décline en définitions variées dans les recherches en psychologie et en psychopédagogie. Notre conception du bien-être articule des aspects associés au bien-être psychologique et subjectif. À l'instar de plusieurs chercheurs (p ex., Keyes, Shmotkin et Ryff, 2002), nous percevons ces orientations de recherche de façon complémentaire.

Alors que certains auteurs associent le bien-être au plaisir et au bonheur (Diener et Lucas, 1999; Laguardia et Ryan, 2000), d'autres ciblent plutôt les manifestations liées à un bon fonctionnement, comme les relations interpersonnelles positives, un certain contrôle sur sa vie et des capacités d'adaptation (Cowen, 1994). Le bien-être 
peut également être associé à la santé mentale positive (Consortium conjoint pour les écoles en santé, 2010). Enfin, d'autres associeront le bien-être psychologique à la prévalence des facteurs de protection sur les facteurs de risque, en ne le limitant pas à l'absence de psychopathologie (Morrison, Kirby, Losier et Allain, 2009).

Selon nous, le concept de bien-être est multidimensionnel et combine tant la perception subjective de l'individu sur son état général que l'engagement de celui-ci dans sa croissance personnelle. Le bien-être fait donc référence au plaisir, au bonheur vécu et aux capacités d'adaptation de l'individu. Ces capacités peuvent être liées à son sentiment de contrôle sur sa vie et aux relations interpersonnelles positives qu'il entretient avec les gens qui l'entourent, ainsi qu'à sa santé mentale.

\section{Sentiment d'appartenance à l'école}

Le sentiment d'appartenance à l'école contribue au développement social, personnel, physique et mental de l'élève; il serait également un facteur de protection en ce qui concerne les comportements à risque (Clement, 2010) en plus d'être lié au bien-être chez les jeunes (Correa-Velez et al., 2010; Kia-Keating et Ellis, 2007). Dans cette recherche, nous avons considéré le sentiment d'appartenance comme un concept multidimensionnel qui peut être associé au sentiment vécu d'acceptation, de respect, d'inclusion et de soutien de son environnement (Goodenow, 1993). Nous nous appuyons aussi sur la conceptualisation de St-Amand (2015), qui comporte quatre dimensions: émotionnelle, sociale, participative et adaptative. Cet auteur dégage quatre grandes catégories d'attributs définitionnels associés au vécu de l'élève: 1) l'élève doit ressentir une émotion positive à l'égard du milieu scolaire; 2) l'élève doit entretenir des relations sociales de qualité avec les membres du milieu scolaire; 3) l'élève doit s'impliquer activement dans les activités de la classe ou celles de l'école; 4) l'élève doit percevoir une certaine synergie (harmonisation), voire une similarité avec les membres de son groupe (St-Amand, 2015, p. 57).

Pour cette recherche, nous ciblons les dimensions émotionnelle et sociale. Selon la recension de St-Amand (2015) reprenant les propos de Goodenow (1993) et de William et Downings (1998), les rapports sociaux liés au sentiment d'appartenance peuvent être associés aux encouragements, à la valorisation, à l'acceptation, au soutien, au respect et à l'amitié. Nous considérons également, de façon secondaire, la dimension participative, qui renvoie à l'implication active des élèves dans les activités de la classe ou dans celles de l'école, et la dimension adaptative, qui renvoie à la capacité de l'élève d'harmoniser ses besoins et ses désirs avec ceux des personnes constituant son groupe (St-Amand, 2015). 


\section{L’approche systémique}

Notre recherche-action s'inscrit dans une approche systémique qui considère le jeune réfugié se développant dans un contexte, dans un temps particulier et en fonction de ses caractéristiques personnelles. Basée sur les travaux de Bronfenbrenner (1995), cette approche nous incite à porter une attention particulière à l'interinfluence entre l'individu et son environnement. Sans entrer dans les détails de ce modèle, nous considérons le bien-être, le sentiment d'appartenance à l'école, le vécu et les caractéristiques du jeune réfugié pour représenter l'individu, élément central de la théorie bioécologique. Nous prenons également en considération des éléments indirects de contexte tels que le milieu scolaire, mais aussi tous les aspects culturels ainsi que toutes les valeurs et les normes qui pourraient influencer et être influencés par les aspects individuels à l'étude.

À la lumière de ces précisions, nous poursuivons deux objectifs spécifiques: 1) documenter l'influence de l'action sur le développement du bien-être, le sentiment d'appartenance à l'école ainsi que sur l'expérience scolaire de ces élèves; et 2) documenter l'implantation d'une action (modalités, déroulement, conditions facilitantes, obstacles, conséquences institutionnelles) en vue d'acquérir une compréhension approfondie des modifications apportées sur le plan des pratiques dans les écoles partenaires.

Dans cet article, nous analysons le corpus de données recueillies dans une perspective systémique, considérant le contexte particulier de l'accueil des réfugiés syriens en 2016-2017, qui souligne l'influence de la gouvernance scolaire et de la formation du personnel enseignant sur le bien-être et le sentiment d'appartenance de ces jeunes.

\section{MÉTHODOLOGIE}

En cohérence avec le caractère exploratoire de nos objectifs de recherche, nous avons mené une recherche-action qualitative visant l'amélioration des pratiques, l'engagement individuel et collectif des acteurs impliqués, et une démarche de recherche adoptant ce cycle: planification, action, observation, réflexion (Morrissette, 2013).

\section{L'action: les groupes de parole}

L'action se déroule dans deux écoles primaires et une école secondaire ayant des points de service pour l'accueil des réfugiés syriens à Laval et à Montréal. Nous proposons des groupes de parole sur des sujets sensibles: le voyage, la migration, la mort et les pertes, les différences, la foi, la violence, l'identité, la famille, la vie et l'expérience des groupes de parole. Lors des rencontres, une traduction arabe est fournie, et l'enseignante ou l'enseignant participe à l'activité. 
Les groupes de parole s'inscrivent dans une approche d'intervention psychopédagogique et psychosociale en milieu scolaire, et ils offrent un cadre propice à la rencontre humaine et à l'expression symbolique nécessaires pour la promotion de la santé mentale. Cette activité se base sur le principe clinique spécifiant qu'un espace de parole libre, dans lequel les élèves peuvent discuter de leurs expériences et de leurs sentiments, favorise le développement de leur bien-être et celui de leur sentiment d'appartenance à l'école (Papazian-Zohrabian et al., 2017).

\section{Les participantes et les participants}

Les participantes et les participants à cette recherche-action sont les élèves, les enseignants des classes d'accueil, ainsi que les directions de ces établissements. Il s'agit d'environ 35 élèves du primaire (deux classes), âgés de 6 à 11 ans, et de 48 élèves du secondaire (trois classes) âgés de 13 à 17 ans. À cela s'ajoute cinq enseignants titulaires des classes d'accueil participantes et trois directeurs adjoints (un par école).

La participation des élèves consiste à assister aux groupes de parole, et celle des acteurs scolaires consiste à réaliser des entrevues individuelles ou en groupe avant et après l'action.

\section{La collecte des données}

La collecte de données est réalisée grâce à l'enregistrement audio: 1) des entrevues individuelles ou de groupe précédant l'action (directions, personnel enseignant des classes d'accueil); 2) des séances des groupes de parole menés avec les jeunes et leur enseignante ou leur enseignant; et 3 ) des entrevues semi-dirigées après l'action (directions et personnel enseignant).

Des informations relatives au déroulement de l'activité ainsi que des éléments contextuels sont recueillis dans les journaux de bord des chercheurs, et ces données nuancent l'analyse thématique réalisée à l'aide du logiciel QDA Miner.

\section{RÉSULTATS}

L'analyse de ces données nous permet de dégager deux catégories de résultats selon nos objectifs spécifiques: ceux en lien avec le développement du bien-être et du sentiment d'appartenance des élèves réfugiés syriens, et ceux décrivant les conditions favorisant ou non le développement du bien-être et du sentiment d'appartenance lors de l'accueil chez les élèves réfugiés syriens. Dans cet article, nous 
présentons seulement les résultats en lien avec le parcours postmigratoire des élèves participants ${ }^{1}$.

\section{Mal-être à l'école secondaire et discrimination}

Lors des groupes de parole, les élèves des classes du secondaire ont partagé des expériences difficiles vécues avec les élèves des classes ordinaires, telles les moqueries, la violence verbale, les situations d'intimidation, voire de racisme, ou les difficultés à s'intégrer aux autres élèves. Ces situations semblent témoigner d'un climat tendu résultant de la division entre les élèves de la classe d'accueil et ceux des classes ordinaires.

Dans les témoignages des élèves, nous notons une discrimination liée à l'identité ethnique: «Je dis que c'est pas juste les [membres d'une autre communauté arabe] qui détestent les Syriens, c'est le tout» (élève du secondaire) et une discrimination liée au contexte scolaire: «[...] nous sommes dans les accueils, nous sommes... avec le temps, habitués à des mots comme ça [en référence à l'expression "les accueils"] ou des mots qui nous insultent comme ça» (élève du secondaire).

Les divers témoignages des participantes et des participants posent aussi la question de la surveillance des espaces communs à l'école et la gestion des situations conflictuelles. Plusieurs situations de violence ou d'abus auraient lieu dans ces endroits. Selon les paroles des enfants, ces incidents sont récurrents et semblent également créer un mal-être chez certains élèves.

«Une fois, ils étaient devant leur casier, tous les Syriens, la cloche a sonné. Donc, il y avait un groupe de garçons [d'une autre communauté arabe] en face et ils jetaient des spaghettis sur les Syriens. [...] Ils ont versé des jus aussi sur leur tête» (traductrice reprenant les propos d'un élève du secondaire).

\section{Faible sentiment d'appartenance à l'école}

Pour ce qui est de l'influence de l'activité sur le sentiment d'appartenance à l'école, le fait de pouvoir partager les émotions vécues semble plutôt avoir renforcé les liens intragroupes et alimenté la cohésion du groupe classe: «[Dans le groupe,] tout le monde est là pour chaque personne» (élève du secondaire).

Les élèves apprennent à communiquer, à être authentiques les uns avec les autres et à mieux se connaître: «J'aime beaucoup, c'est la première fois qu'on se parle avec les

1. Nous avons spécifié dans le texte les thèmes abordés uniquement par les élèves du secondaire. 
amis. Maintenant, je les connais plus, et avec le temps, on va les connaître de plus en plus» (élève du primaire).

Toutefois, les élèves ne semblent pas avoir développé de sentiment d'appartenance à l'école; celui-ci semble au contraire très faible. L'écart entre les élèves des classes ordinaires et ceux des classes d'accueil semble y avoir contribué. Cette réalité est également perceptible entre les élèves de la classe d'accueil et ceux des classes ordinaires issus de l'immigration.

«Ici, à l'école, il y a beaucoup de personnes, pas juste québécoises, il y a de l'intimidation...» (élève du secondaire).

Cette division entre la classe d'accueil et les classes ordinaires se vit même parfois parmi les acteurs scolaires: «L'accueil, c'est comme une école dans l'école, un petit groupe fermé» (acteur scolaire).

\section{Intégration sociale difficile}

Le mal-être des élèves réfugiés syriens ainsi que leur faible sentiment d'appartenance à l'école semble renforcé par le contexte scolaire. Le personnel de l'école partage cette perception. Certains vont même jusqu'à affirmer que l'intégration est un échec en l'expliquant par l'homogénéité ethnoculturelle des élèves réfugiés syriens.

«Ces élèves sont toujours à part. C'est un échec qu'on vit. C'est pas un bon exemple de comment intégrer des élèves. Ils sont tellement homogènes - ils viennent du même coin, des fois de la même rue - qu'on ne peut pas les intégrer. Ils ne sont pas intéressés aux autres, ils ne parlent qu'entre eux autres, qu'arabe; l'intégration ne fonctionne pas ici» (acteur scolaire).

\section{Difficultés organisationnelles liées à l'arrivée rapide et importante des réfugiés syriens}

Plusieurs facteurs organisationnels sont à la base de cette difficulté d'accueil des élèves réfugiés. Les locaux disponibles étant le critère principal pour recevoir ces élèves dans les écoles, des classes d'accueil ont été ouvertes sans qu'il y ait de «culture d'accueil» dans l'établissement. Ce manque, tant auprès du personnel scolaire que des élèves, a contribué au développement de l'écart entre les élèves réfugiés syriens et les élèves des classes ordinaires.

«On n’a jamais eu d'accueil, ça aide pas» (acteur scolaire). 
La rigidité de l'organisation scolaire, des modèles de services d'accueil et de francisation ainsi que des procédures d'intégration des élèves de l'accueil aux classes ordinaires est aussi ressortie.

Dans les écoles participantes, le modèle de services offert est la classe d'accueil fermée, avec quelques intégrations lors d'activités parascolaires. Selon les participantes et les participants, cette situation nuit aux élèves, car ceux-ci sont rarement en relation avec les élèves des classes ordinaires. De plus, la transition de l'accueil aux classes ordinaires semble complexe: les membres du personnel enseignant de l'accueil hésitent à laisser partir «leurs élèves » et ceux des classes ordinaires ont peur que les élèves soient trop faibles en français pour réussir.

"Je pense que les profs jouent un rôle; au lieu d'être rassembleurs et de favoriser l'intégration, ils les retiennent. C'est à moi, mes élèves, mes petits poussins» (acteur scolaire).

Ces «nouvelles classes» étaient constituées d'une grande majorité d'élèves syriens. Ces groupes homogènes ont déstabilisé certains acteurs scolaires. Habitués aux groupes hétérogènes dans lesquels la langue commune est le français, ce groupe d'élèves provenant souvent des mêmes endroits, partageant tous la même langue (arabe) et parfois même se connaissant déjà, a amené les membres du personnel enseignant à moins bien connaître leurs élèves et à se sentir déstabilisés malgré leur expérience.

«[...] j'ai l'impression que je connais mal mes élèves. Même si je suis titulaire de classe, avec mon expérience, on connaît assez bien nos élèves» (acteur scolaire).

Par ailleurs, l'enjeu du classement des élèves est très important. Plusieurs parents veulent que leurs enfants passent rapidement aux classes ordinaires, et certains élèves aussi. Toutefois, les procédures et les critères pour intégrer les classes ordinaires semblent flous et subjectifs.

«Parce qu'il y a eu énormément d'irrégularités... l'année passée, tout était possible [allusion à la première année d'accueil]» (acteur scolaire).

\section{Manque de ressources}

En outre, plusieurs participantes et participants notent un manque de ressources humaines (p. ex., un manque de professionnels, surtout de psychologues scolaires pouvant intervenir auprès des jeunes et de leurs familles) et matérielles (p. ex., un manque de dictionnaires, de manuels, de budget pour les sorties). 
«[...] psychologie, des services comme ça, c'est tellement rare. Ça pas été prévu. Donc, de vous voir arriver, ça comblait un besoin» (acteur scolaire).

«Les groupes de discussion, c'est pas miraculeux, il y a des enfants qui n'ont pas parlé... On a besoin de plus... Il n'y en a pas, de ressources psychologiques» (acteur scolaire).

\section{Manque de formation sur la situation et le vécu des réfugiés}

Nous avons remarqué la difficulté des acteurs scolaires à reconnaître les signes de mal-être chez les jeunes, une confusion entre certaines problématiques de santé mentale et les traumatismes, une méconnaissance des particularités de la situation des réfugiés en général et des réfugiés syriens en particulier, et une banalisation de leur situation.

«Plus j'apprends à les connaître, plus on apprend que, je ne dis pas qu'ils n'ont pas souffert, mais ils n'ont pas traversé une énorme crise au point de vue que nous, les Occidentaux, on voit, mon Dieu, les bombes, etc. Ils ont quitté rapidement vers le Liban, ont été scolarisés, viennent de familles généralement bien nanties, ils avaient des domestiques. Oui, ils ont dû abandonner des biens matériels là-bas, oui, ils ont vécu un déracinement, mais ils n'ont pas manqué de grand-chose. Oui, en arrivant ici, ils ont vécu un choc, mais je ne pense pas que c'est un choc traumatique, mais je ne suis pas psychologue...» (acteur scolaire).

Or, les groupes de parole ont permis à ces jeunes de symboliser de multiples pertes et des traumas méconnus des acteurs scolaires.

L'analyse des entrevues pré et postaction menées auprès de ces derniers nous a montré que plusieurs d'entre eux ne connaissent pas les interventions psychosociales possibles en milieu scolaire ou même l'importance ou la manière d'aborder le vécu des élèves. Certains confondent l'expression des émotions et du vécu avec le développement du lexique relatif aux émotions: "On a parlé du vocabulaire des sentiments, on a fait un petit truc sur comment tu te sens aujourd'hui, mais on n'a pas plus élaboré que ça» (acteur scolaire). D’autres ne connaissent pas les difficultés d'apprentissage dues aux deuils et aux traumatismes. Cette ignorance peut mener à une attitude empreinte de jugement envers les élèves.

«Il y en a qui s'intègrent et il y en a que non, et on ne sent pas une volonté [de vouloir s'intégrer] [...] Si, à ce point-ci de l'année, tu sais pas plus parler français qu'au début de l'année, c'est que tu as une volonté de... il y a pas juste une question d'apprentissage, des capacités ou quoi que ce soit, il y a une volonté, à ce temps-ci de l'année. Il y en a qui ne veulent pas» (acteur scolaire). 


\section{Manque de formation en éducation interculturelle}

Dans le cadre des groupes de parole, de nombreux élèves nous ont parlé de situations de «quiproquo culturel» et de la mauvaise interprétation des acteurs scolaires de certains codes culturels.

«En Syrie ou bien au Liban, quand quelqu'un, un garçon coupe ses cheveux, on lui donne un [mot arabe] c'est une belle coupe, on fait comme ça [tape derrière la nuque]. Ici, quand quelqu'un coupe ses cheveux, on fait ça [...], un surveillant comprend pas» (élève du secondaire).

Bien que les acteurs scolaires soient conscients de l'importance du recours à la langue maternelle dans l'apprentissage d'une langue seconde, dans ce cas-ci, l'homogénéité des groupes, la prédominance de la langue arabe comme langue de communication entre les élèves ainsi que la difficulté d'imposer la langue d'enseignement viennent «chercher» les acteurs dans leur identité québécoise.

«Les Québécois de souche, on ne parle pas l'arabe, alors moi je me sens exclue, ils savent que moi, je me sens exclue dans la classe... je ne peux pas communiquer avec vous, j'ai ben de la misère...» (acteur scolaire).

Cette perception et cette réaction polarisent le lien éducatif, et elles entravent la réelle rencontre entre les élèves et les acteurs scolaires. Or, de nombreux élèves réfugiés syriens désirent communiquer en français, mais avec qui?

«[...] j'ai beaucoup d'amis, mais je veux avoir des amis québécois, je veux pratiquer avec lui, quand je vois un ami arabe, je ne peux pas lui parler en français » (élève du secondaire).

Cette homogénéité du groupe nourrit une perception stigmatisante chez certains acteurs, et les groupes de parole sont venus nuancer cette perception réductrice.

«[...] on voit les différences entre les élèves, j’ai vu beaucoup de différences sur leur façon d'être et de voir les choses. Je pensais que ce serait les Syriens, mais chaque élève avait ses opinions différentes» (acteur scolaire).

L'analyse de ces résultats nous amène à cerner les défis de l'accueil des élèves réfugiés et à réfléchir sur les enjeux pour les milieux scolaires.

\section{DISCUSSION}

Le point de départ de notre recherche était la situation psychosociale des réfugiés et le potentiel de mal-être dans lequel ils se trouvent à leur arrivée dans un pays 
d'accueil. Nous pensions que cette situation pouvait défavoriser leur expérience scolaire et influencer négativement leur accueil. L'analyse de nos données nous a cependant amenés à dégager surtout certaines problématiques postmigratoires importantes mettant à rude épreuve l'expérience scolaire des élèves réfugiés: malêtre et discrimination à l'école, faible sentiment d'appartenance à l'école, intégration sociale difficile, difficultés organisationnelles, manque de ressources, lacunes dans la formation des acteurs scolaires.

Nos résultats rejoignent ceux de Montgomery et Foldspang (2008) soulignant l'importance de l'influence de facteurs postmigratoires (famille, amis, relations sociales et services) sur la santé mentale des jeunes réfugiés. À l'instar d'autres chercheurs, nous avons vu le rôle important de l'école dans l'adaptation, le bien-être et l'intégration des jeunes réfugiés (Bartlett et al., 2017; Block et al., 2014; Hamilton et Moore, 2004; Kirk, 2002; Kupzyk et al., 2016; Persson et Rousseau, 2008; Sarr et Mosselson, 2010; Stewart, 2011). Ainsi, l'attitude des acteurs scolaires, leurs liens avec les jeunes, les relations positives qu'ils favorisent, le sentiment de sécurité qu'ils assurent et les activités qu'ils proposent ont tous une influence positive sur le développement des jeunes. Toutefois, alors que les jeunes immigrants qui fréquentent l'école québécoise s'auto-identifient rarement d'un point de vue ethnoculturel (Kanouté et al., 2016), les exemples de violence et de discrimination relevés dans cette recherche dénotent que l'identification ethnoculturelle des membres du milieu d'accueil (élèves et acteurs scolaires) peut devenir discriminatoire et entraîner une scission entre les groupes. Pour désamorcer ces phénomènes systémiques et préparer les écoles à un meilleur accueil des élèves réfugiés, il est important d'être conscients de certains enjeux relatifs à la gouvernance et à la formation des acteurs scolaires.

\section{Enjeux pour la gouvernance scolaire}

L'expérience scolaire des élèves immigrants et réfugiés peut être favorisée par certaines initiatives de la direction, telles que l'organisation de diverses activités permettant la rencontre des élèves des classes d'accueil et ordinaires, la surveillance des zones de convivialité ainsi que la lutte contre la discrimination et le racisme. Le fossé relevé entre les élèves des classes d'accueil et ceux des classes ordinaires ainsi que les situations de violence et de discrimination que les jeunes relatent dans le cadre de cette recherche soulignent l'importance d'une gestion adéquate de ces situations et de ces zones. Divers auteurs (Astor, Meyer et Pitner, 2001; Langhout et Annear, 2010) abordent le sujet des «zones scolaires communes» telles que la cafétéria, les classes, les corridors ou les casiers, qui peuvent être perçues par les élèves comme étant plus ou moins sécuritaires.

Par ailleurs, les critères et les procédures d'intégration des élèves dans les classes ordinaires sont à revoir. Certains acteurs scolaires interrogés pour cette étude mettent en relief les incohérences et les irrégularités des modèles en ce qui concerne le passage 
à la classe ordinaire. Ces résultats peuvent faire écho aux résultats d'autres études telles que celle menée par De Koninck et Armand (2012) faisant état des différences entre les modèles d'intégration des élèves allophones dans les écoles québécoises et mettant l'accent sur l'importance, pour les acteurs scolaires, de connaître les droits et les besoins des élèves et d'adapter les modèles à ces besoins.

Le manque de ressources humaines et matérielles a aussi fréquemment été relevé. Certains auteurs montrent l'importance des interventions psychosociales pour la promotion de la santé mentale des réfugiés (Silove et al., 2017). Ce genre d'intervention en milieu scolaire nécessiterait une nouvelle organisation des ressources professionnelles, un soutien constant au personnel enseignant ainsi que des formations générales pour l'équipe-école.

\section{Enjeux pour la formation des intervenantes et des intervenants scolaires}

Une étude de Rossiter et Rossiter (2009), citée par Hadfield, Ostrowski et Ungar (2017), souligne que la formation du personnel enseignant canadien est limitée pour proposer aux jeunes réfugiés un milieu social et éducatif culturellement adéquat pour répondre à leurs besoins. Nos résultats mettent en évidence les besoins en formation sur la situation des réfugiés, leur santé mentale, l'influence des deuils et des traumatismes sur l'adaptation et les apprentissages des jeunes, les signes de mal-être chez les jeunes et les pistes d'intervention en milieu scolaire. La formation des milieux éducatifs en santé mentale est pertinente, entre autres pour connaître les ressources de soutien et pour clarifier le rôle des intervenantes et des intervenants (Papazian-Zohrabian, 2015). Selon les études consultées, les membres du personnel enseignant perçoivent la responsabilité de l'école pour le soutien de la santé mentale des élèves (Reinke, Stromont, Herman, Puri et Goel, 2011). Ils soulignent le fait que leurs connaissances en santé mentale ne sont pas suffisantes pour aborder ces problèmes et pour agir de façon appropriée (Kirby et Keon, 2006; Mazzer et Rickwood, 2015; Reinke et al., 2011).

L'éducation interculturelle doit aussi faire l'objet de formations dans les milieux scolaires. Selon Steinbach (2015), qui considère que l'intégration est bidirectionnelle et que les nouveaux arrivants comme les membres de la société d'accueil y participent, il est pertinent d'informer les acteurs scolaires et les élèves des attitudes et des comportements qui peuvent favoriser les interactions interculturelles positives et l'intégration des élèves immigrants. C'est d'ailleurs l'une des orientations de la politique d'accueil et d'intégration scolaire du Québec (Ministère de l'Éducation et de l'Enseignement supérieur, 2017).

Dans le contexte actuel, où l'accueil des réfugiés se heurte parfois à une atmosphère de polarisations sociales, les milieux scolaires se trouvent devant les défis de la 
promotion du bien-être psychologique des jeunes réfugiés et de leur sentiment d'appartenance à l'école.

\section{FORCES ET LIMITES}

Notre recherche a permis de donner la parole aux jeunes réfugiés syriens, à leurs enseignantes et enseignants, ainsi qu'aux directions de leurs écoles, et de mettre en relief les multiples besoins et défis des écoles dans le processus de l'accueil des élèves immigrants de façon générale. Les élèves ont parlé dans un contexte d'expression libre - les groupes de parole -, où juste une thématique leur a été proposée. Les acteurs scolaires ont été amenés à verbaliser leurs difficultés et leurs besoins. Les résultats de notre étude ne peuvent pas être généralisés à toutes les écoles québécoises, étant donné le nombre restreint de participants. Toutefois, ceux-ci nous permettent de mieux comprendre la réalité des élèves, du personnel enseignant et des directions d'école à l'égard des défis de l'accueil des élèves réfugiés en contexte postmigratoire.

\section{CONCLUSION}

Sachant que les expériences scolaires positives servent de facteurs de protection pour les jeunes élèves réfugiés (Fazel, Garcia et Stein, 2016), il est d'une grande importance de répondre aux besoins des milieux scolaires afin d'optimiser l'accueil et l'expérience scolaire de ces élèves. L'urgence de penser la gouvernance scolaire et la formation des acteurs scolaires se fait sentir. La recherche-action menée auprès des élèves réfugiés syriens répond ponctuellement aux besoins de ces jeunes. Elle met en valeur la nécessité de développer un bon climat scolaire et des pratiques adéquates. Elle suggère également l'importance de promouvoir les recherches et les actions dans le domaine de l'éducation en contexte de diversité.

\section{Références bibliographiques}

ASTOR, R. A., MEYER, H. A. et PITNER, R. O. (2001). Elementary and middle school students'perceptions of violence-prone school subcontexts. Elementary School Journal, 101(5), 511-528.

BARTLETT, L., MENDENHALL, M. et GHAFFAR-KUCHER, A. (2017). Culture in acculturation: Refugee youth's schooling experiences in international schools in New York City. International Journal of Intercultural Relations, 60, 109-119. 
BEISER, M. et HOU, F. (2016). Mental health effects of premigration trauma and postmigration discrimination on refugee youth in Canada. The Journal of Nervous and Mental Disease, 204(6), 464-470.

BLOCK, K., CROSS, S., RIGGS, E. et GIBBS, L. (2014). Supporting schools to create an inclusive environment for refugee students. International Journal of Inclusive Education, 18(12), 1337-1355.

BRONFENBRENNER, U. (1995). Developmental ecology through space and time. Dans P. Moen, G. H. Elder et K. Lüscher (dir.), Examining lives in context: Perspectives on the ecology of human development (p. 619-648). Washington, DC: American Psychological Association.

CLEMENT, N. (2010). Student wellbeing at school: The actualization of values in education. Dans T. Lovat, R. Toomey et E. Clement (dir.), International research handbook on values education and student wellbeing. Dordrecht, Pays-Bas: Springer.

Consortium conjoint pour les écoles en santé. (2010). Le milieu scolaire comme terrain propice à la promotion de la santé mentale positive: meilleures pratiques et perspectives. Repéré à https://www.jcsh-cces.ca/upload/PMH_French_Nov\%2016_ WebReady_1.pdf

CORREA-VELEZ, I., Gifford, S. M. et Barnett, A. G. (2010). Longing to belong: Social inclusion and wellbeing among youth with refugee backgrounds in the first three years in Melbourne, Australia. Social Science \& Medicine, 71(8), 1399-1408.

COWEN, E. L. (1994). The enhancement of psychological wellness: Challenges and opportunities. American Journal of Community Psychology, 22(2), 149-179.

DE KONINCK, Z. et ARMAND, F. (2012). Entre métropole et régions, un même raisonnement peut-il soutenir un choix de modèles de services différent pour l'intégration des élèves allophones? Diversité urbaine, 12(1), 69-85.

DIENER, E. et LUCAS, R. E. (1999). Personality and subjective well-being. Dans D. Kahneman, E. Diener et N. Schwarz (dir.), Well-being: Foundations of hedonic psychology. New York, NY: Russel Sage Foundation.

FAZEL, M., GARCIA, J. et STEIN, A. (2016). The right location? Experiences of refugee adolescents seen by school-based mental health services. Clinical Child Psychology and Psychiatry, 21(3), 368-380.

GOODENOW, C. (1993). The psychological sense of school membership among adolescents: Scale development and educational correlates. Psychology in the Schools, 30(1), 79-90. 
HADFIELD, K., OSTROWSKI, A. et UNGAR, M. (2017). What can we expect of the mental health and well-being of Syrian refugee children and adolescents in Canada? Canadian Psychology/Psychologie canadienne, 58(2), 194-201.

HAMILTON, R. et MOORE, D. (2004). Education of refugee children: Documenting and implementing change. Dans R. Hamilton et D. Moore (dir.), Educational interventions for refugee children (p. 106-116). New York, NY: Routledge.

HART, R. (2009). Child refugees, trauma and education: Interactionist considerations on social and emotional needs and development. Educational Psychology in Practice, 25(4), 351-368.

HASSAN, G., KIRMAYER, L. J., VENTEVOGEL, P., MEKKI BERRADA, A., QUOSH, C., El CHAMMAY, R. ...et SONG, S. (2016). Culture, contexte du conflit, santé mentale et bien-être psychosocial des Syriens. Rapport sur la santé mentale et le soutien psychosocial à l'intention du personnel travaillant auprès des Syriens touchés par le conflit armé. Genève, Suisse: Haut Commissariat des Nations Unies pour les réfugiés.

Haut Commissariat des Nations Unies pour les réfugiés, Canada. (2017). Refugee resettlement facts. Repéré à http://www.unhcr.ca/wp-content/uploads/2017/04/ Canadian-Resettlement-Fact-Sheet-ENG-April-2017.pdf

KANOUTÉ, F, GOSSELIN-GAGNÉ, J., GUENNOUNI HASSANI, R. et GIRARD, C. (2016). Points de vue d'élèves issus de l'immigration sur leur expérience socioscolaire en contexte montréalais défavorisé. Alterstice, 6(1), 13-25.

KANOUTÉ, F. et LAFORTUNE, G. (2011). Familles québécoises d'origine immigrante. Les dynamiques de l'établissement. Montréal, Québec: Presses de l'Université de Montréal.

KEYES, C. L., SHMOTKIN, D. et RYFF, C. D. (2002). Optimizing well-being: The empirical encounter of two traditions. Journal of Personality and Social Psychology, 82(6), 1007-1022.

KIA-KEATING, M. et ELLIS, B. H. (2007). Belonging and connection to school in resettlement: Young refugees, school belonging, and psychosocial adjustment. Clinical Child Psychology and Psychiatry, 12(1), 29-43.

KIRBY, M. J. et KEON, W. J. (2006). Out of the shadows at last: Transforming mental health, mental illness and addiction services in Canada. Repéré à https://www. mentalhealthcommission.ca/sites/default/files/out_of_the_shadows_at_last_-_full_0 - $0 . p d f$ 
KIRK, J. (2002). Les enfants touchés par la guerre dans les écoles de Montréal. Repéré à www.cgtsim.qc.ca/images/documents/enfants_guerre.pdf

KUPZYK, S. S., BANKS, B. M. et CHADWELL, M. R. (2016). Collaborating with refugee families to increase early literacy opportunities: A pilot investigation. Contemporary School Psychology, 20(3), 205-217.

LAGUARDIA, J. G. et RYAN, R. M. (2000). Buts personnels, besoins psychologiques fondamentaux et bien-être: théorie de l'autodétermination et applications. Revue québécoise de psychologie, 21(2), 281-304.

LANGHOUT, R. D. et ANNEAR, L. (2010). Safe and unsafe school spaces: Comparing elementary school student perceptions to common ecological interventions and operationalizations. Journal of Community and Applied Social Psychology, 21(1), 71-86.

MAZZER, K. R. et RICKWOOD, D. J. (2015). Teachers' role breadth and perceived efficacy in supporting student mental health. Advances in School Mental Health Promotion, 8(1), 29-41.

McNEELY, C. A., MORLAND, L., DOTY, S. B., MESCHKE, L. L., AWAD, S., HUSAIN, A. et NASHWAN, A. (2017). How schools can promote healthy development for newly arrived immigrant and refugee adolescents: Research priorities. Journal of School Health, 87(2), 121-132.

Ministère de l'Éducation et de l'Enseignement supérieur. (2017). Soutien au milieu scolaire 2017-2018. Intégration et réussite des élèves issus de l'immigration et éducation interculturelle. Repéréàhttp://www.education.gouv.qc.ca/fileadmin/site web/documents/education/diversite/Guide-soutien-_milieu-scolaire_2017-2018.pdf

Ministère de l'Immigration, de la Diversité et de l'Inclusion. (2017). Bienvenue aux réfugiés syriens. Repéré à http://www.immigration-quebec.gouv.qc.ca/fr/ informations/accueil-refugies-syriens

MONTGOMERY, E. et FOLDSPANG, A. (2008). Discrimination, mental problems and social adaptation in young refugees. European Journal of Public Health, 18(2), 156-161.

MORRISON, W., KIRBY, P., LOSIER, G. et ALLAIN, M. (2009). Conceptualizing psychological wellness: Addressing mental fitness needs. Journal of the Canadian Association of Principals, 17(2), 19-21.

MORRISSETTE, J. (2013). Recherche-action et recherche collaborative: quel rapport aux savoirs et à la production de savoirs? Nouvelles pratiques sociales, 25(2), 35-49. 
ÖZER, S., IRIN, S. et OPPEDAL, B. (2013). Bahçe ehir study of Syrian refugee children in Turkey. Repéré à www.fhi.no/dokumenter/c83Fb3a78c.pdf

PACIONE, L., MEASHAM, T., KRONICK, R., MELONI, F., RICARD-GUAY, A., ROUSSEAU, C. et RUIZ-CASARES, M. (2012). The mental health of children facing collective adversity: Poverty, homelessness, war and displacement. Dans J. M. Rey (dir.), IACAPAP Textbook of child and adolescent mental health (p. 1-35). Genève, Suisse: International Association for Child and Adolescent Psychiatry and Allied Professions.

PAPAZIAN-ZOHRABIAN, G. (2015). Les enfants traumatisés et endeuillés par la guerre. Dans C. Fawer Caputo et M. Julier-Costes (dir.), La mort à l'école: annoncer, accueillir, accompagner (p. 249-270). Bruxelles, Belgique: De Boeck Supérieur

PAPAZIAN-ZOHRABIAN, G. (2016). Les jeunes réfugiés et les enfants de la guerre à l'école québécoise. Dans M. Potvin, M.-O. Magnan et J. Larochelle-Audet (dir.), La diversité ethnoculturelle, religieuse et linguistique en éducation: théorie et pratique (p. 183-196). Québec, Québec: Fides.

PAPAZIAN-ZOHRABIAN, G., LEMIRE, V., MAMPRIN, C., TURPIN-SAMSON, A. et AOUN, R. (2017). Mener des groupes de parole en contexte scolaire. Guide pour les enseignants et les professionnels. Montréal, Québec: Centre d'intervention pédagogique en contexte de diversité de la Commission scolaire MargueriteBourgeoys et Université de Montréal.

PERSSON, T. J. et ROUSSEAU, C. (2008). School-based interventions for minors in war-exposed countries: A review of targeted and general programmes. Torture: Quarterly Journal on Rehabilitation of Torture Victims and Prevention of Torture, 19(2), 88-101.

REINKE, W. M., STROMONT, M., HERMAN, K. C., PURI, R. et GOEL, N. (2011). Supporting children's mental health in schools: Teacher perceptions of needs, roles, and barriers. School Psychology Quarterly, 26(1), 1-13.

RUTTER, J. (2003). Supporting refugee children in $21^{\text {st }}$ century Britain: A compendium of essential information. Stoke on Trent, Angleterre: Trentham Books.

SAROT, A. et MORO, M. R. (2016). L'école, première institution d'accueil de l'altérité: pour une société inclusive. Le sujet dans la cité, 2(7), 65-76.

SARR, K. G. et MOSSELSON, J. (2010). Issues in teaching refugees in U.S. schools. Yearbook of the National Society for the Study of Education, 109(2), 548-570. 
SILOVE, D., VENTEVOGEL, P. et REES, S. (2017). The contemporary refugee crisis: An overview of mental health challenges. World Psychiatry, 16(2), 130-139.

SIRIN, S. R. et ROGERS-SIRIN, L. (2015). The educational and mental health needs of Syrian refugee children. Washington, DC: Migration Policy Institute.

ST-AMAND, J. (2015). Le sentiment d'appartenance à l'école: un regard conceptuel, psychométrique et théorique (Thèse de doctorat, Université de Montréal, Canada). Repéré à https://papyrus.bib.umontreal.ca/xmlui/bitstream/handle/1866/14049/ St-Amand_Jerome_2015_these.pdf?sequence $=2$

STEINBACH, M. (2015). Les défis de l'intégration sociale des jeunes immigrants à l'extérieur de la métropole québécoise. Diversité urbaine, 15(1), 69-85.

STEWART, J. (2011). Supporting refugee children: Strategies for educators. Toronto, Canada: University of Toronto Press.

TYRER, R. A. et FAZEL, M. (2014). School and community-based interventions for refugee and asylum seeking children: A systematic review. PLOS One, 9(2), e89359.

YOHANI, S. (2010). Nurturing hope in refugee children during early years of post-war adjustment. Children and Youth Services Review, 32(6), 865-873. 